\title{
Temperature Variations Analysis for Condensed Matter Micro- and Nanoparticles Combustion Burning in Gaseous Oxidizing Media by DTM and BPES
}

\author{
M. Hatami, ${ }^{1}$ D. D. Ganji, ${ }^{1}$ and K. Boubaker ${ }^{2}$ \\ ${ }^{1}$ Department of Mechanical Engineering, Babol University of Technology, P.O. Box 484, 47148-71167 Babol, Iran \\ ${ }^{2}$ Equipe de Physique des Dispositifs à Semiconducteurs, Faculté des Sciences de Tunis, Campus Universitaire, 2092 Tunis, Tunisia \\ Correspondence should be addressed to K. Boubaker; mmbb11112000@yahoo.fr
}

Received 29 May 2013; Accepted 23 July 2013

Academic Editors: C. Andreani and J. Tempere

Copyright (C) 2013 M. Hatami et al. This is an open access article distributed under the Creative Commons Attribution License, which permits unrestricted use, distribution, and reproduction in any medium, provided the original work is properly cited.

Combustion process for iron particles burning in the gaseous oxidizing medium is investigated using the Boubaker polynomial expansion scheme (BPES) and the differential transformation method (DTM). Effects of thermal radiation from the external surface of burning particle and alterations of density of iron particle with temperature are considered. The solutions obtained using BPES technique and DTM are compared with those of the fourth-order Runge-Kutta numerical method. Results reveal that BPES is more accurate and reliable method than DTM. Also the effects of some physical parameters that appeared in mathematical section on temperature variations of particles as a function of time are studied.

\section{Introduction}

Combustion of metallic particles is one of the most challenging issues in industries that manufacture, process, generate, or use combustible dusts, and an accurate knowledge of their explosion hazards is essential. Many studies have been done for estimating and modeling the particle and dust combustion. Haghiri and Bidbadi [1] investigated the dynamic behavior of particles across flame propagation through a two-phase mixture consisting of micro-iron particles and air. They assumed three zones for flame structure: preheat, reaction, and postflame (burned). Liu et al. [2] analyzed the flame propagation through hybrid mixture of coal dust and methane in a combustion chamber. A one-dimensional, steady-state theoretical analysis of flame propagation mechanism through microiron dust particles based on dust particles' behavior with special remark on the thermophoretic force in small Knudsen numbers is presented by Bidabadi et al. [3]. Haghiri and Bidabadi [4] performed a mathematical model to analyze the structure of flame propagating through a two-phase mixture consisting of organic fuel particles and air. In contrast to previous analytical studies, they take thermal radiation effect into consideration, which has not been attempted before. Recently, Bidbadi and Mafi [5] solved the nonlinear energy equation that resulted from particle combustion modeling by using homotopy perturbation method (HPM), and they presented equations for calculating the convective heat transfer coefficient and burning time for iron particles. Because HPM needs perturbation and a small parameter, it can be solved by other high-accuracy analytical methods where in the present study two of them are presented and compared with fourth-order Runge-Kutta numerical method.

Polynomial expansion methods are extensively used in many mathematical and engineering fields to yield meaningful results for both numerical and analytical analyses. Among the most frequently used polynomials, the Boubaker polynomial expansion scheme which is firstly introduced by Boubacker [6] is one of the interesting tools which were associated with several applied physical and mathematical problems [7-26].

The differential transformation method is an alternative procedure for obtaining an analytic Taylor series solution of differential equations. The main advantage of this method is that it can be applied directly to nonlinear differential 
equations without requiring linearization, discretization, and therefore, it is not affected by errors associated with discretization. The concept of DTM was first introduced by Zhou [27], who solved linear and nonlinear problems in electrical circuits. Chen and Ho [28] developed this method for partial differential equations, and Ayaz [29] applied it to the system of differential equations; this method is very powerful [30]. Jang et al. [31] applied the two-dimensional DTM to partial differential equations. This method was successfully applied to various application problems $[32,33]$.

Motivated by previously mentioned works, this paper aims to introduce two analytical methods for obtaining the temperature of iron particle during combustion. So BPES and DTM are presented. These methods have an excellent agreement with numerical Runge-Kutta method; they also have very low errors without any need for perturbation or discretization compared to previous analytical methods in the literature.

\section{Problem Description}

Consider a spherical particle which due to high reaction with oxygen will be combusted. Since the thermal diffusivity of substance is large and Biot number is small $\left(\mathrm{Bi}_{H} \ll 0.1\right)$, it is assumed that the particle is isothermal. In this state, a lumped system analysis is applicable. When this criterion is satisfied, the variation of temperature with location within the particle will be slight and can be approximated as being uniform, so particle has a spatially uniform temperature, and therefore, the temperature of particle is a function of time only, $T=T(t)$, and is not a function of radial coordinate, $T \neq T(r)$. The assumptions used in this modeling are [5] as follows.

(1) The spherical particle burns in a quiescent, infinite ambient medium, and there are no interactions with other particles, and also the effects of forced convection are ignored.

(2) Thermophysical properties for the particle and ambient gaseous oxidizer are assumed to be constant.

(3) The particle radiates as a gray body to the surroundings without the contribution of the intervening medium.

By these assumptions and considering the particle as a thermodynamic system and by the use of principle of conservation of energy (first law of thermodynamics), the energy balance equation for this particle can be written as

$$
\dot{E}_{\text {in }}-\dot{E}_{\text {out }}+\dot{E}_{\text {gen }}=\left(\frac{d E}{d t}\right)_{p},
$$

where $\dot{E}_{\text {in }}$ is the rate of energy entering the system which is due to absorption of total radiation incident on the particle surface from the surrounding, $\dot{E}_{\text {out }}$ is the rate of energy leaving the system by mechanisms of convection on the particle surface and thermal radiation that emits from the outer surface of particle, $\dot{E}_{\text {gen }}$ is the rate of generation of energy inside the particle due to the combustion process and is equal to the heat released from the chemical reaction, and $(d E / d t)_{p}$ is the rate of change in total energy of particle. These energy terms can be calculated by [5]

$$
\begin{gathered}
\dot{E}_{\text {in }}=\alpha_{s} \sigma A_{s} T_{\text {surr }}^{4}, \\
\dot{E}_{\text {out }}=h_{\text {conv }} A_{s}\left(T_{s}-T_{\infty}\right)+\varepsilon_{s} \sigma A_{s} T_{s}^{4}, \\
\dot{E}_{\text {gen }}=\dot{Q}_{\text {comb }}=\dot{R}_{p} A_{s} \Delta h_{\text {comb }}^{\circ}, \\
\left(\frac{d E}{d t}\right)_{p}=\rho_{p} V_{p} c_{p} \frac{d T_{s}}{d t} .
\end{gathered}
$$

By substituting (2)-(5) in (1),

$$
\begin{aligned}
\alpha_{s} \sigma A_{s} T_{\text {surr }}^{4}-\left(h_{\text {conv }} A_{s}\left(T_{s}-T_{\infty}\right)+\varepsilon_{s} \sigma A_{s} T_{s}^{4}\right) \\
+\dot{R}_{p} A_{s} \Delta h_{\mathrm{comb}}^{\circ} \\
=\rho_{p} V_{p} c_{p} \frac{d T_{s}}{d t}
\end{aligned}
$$

Three reasonable assumptions are used for improving (6).

(I) Both absorptivity and emissivity of the surface depend on the temperature and the wavelength of radiation. Kirchhoff's law of radiation states that the absorptivity and the emissivity of a surface at a given temperature and wavelength are equal $\left(\varepsilon_{s} \simeq \alpha_{s}\right)$.

(II) The initial temperature of the particle at the beginning of combustion can be regarded as the initial condition. This temperature is known as ignition temperature $\left(T(0)=T_{\mathrm{ig}}\right)$.

(III) The density of particle is a function of particle temperature, so it can be considered as a linear function $\left(\rho_{p}=\rho_{p}(T)=\rho_{p, \infty}\left[1+\beta\left(T-T_{\infty}\right)\right]\right)$.

By applying these assumptions, (6) will be converted to the following:

$$
\begin{aligned}
\rho_{p, \infty} & {\left[1+\beta\left(T-T_{\infty}\right)\right] V_{p} c_{p} \frac{d T_{s}}{d t} } \\
& +h_{\text {conv }} A_{s}\left(T_{s}-T_{\infty}\right)+\varepsilon_{s} \sigma A_{s}\left(T_{s}^{4}-T_{\text {surr }}^{4}\right) \\
& -\dot{R}_{p} A_{s} \Delta h_{\mathrm{comb}}^{\circ} \\
= & 0 .
\end{aligned}
$$

For solving this nonlinear differential equation, It is more suitable that all the terms be converted to the 
dimensionless form. The following set of dimensionless variables are defined:

$$
\begin{gathered}
\theta=\frac{T}{T_{\text {ig }}}, \quad \theta_{\infty}=\frac{T_{\infty}}{T_{\text {ig }}}, \\
\theta_{\text {surr }}=\frac{T_{\text {surr }}}{T_{\text {ig }}}, \quad \varepsilon_{1}=\beta T_{\text {ig }}, \\
\tau=\frac{t}{\left(\rho_{p, \infty} V_{p} c_{p} / h_{\text {conv }} A_{s}\right)}, \quad \psi=\frac{\dot{Q}_{\text {comb }}}{h_{\text {conv }} A_{s} T_{\text {ig }}}, \\
\varepsilon_{2}=\frac{\varepsilon_{s} \sigma T_{\text {ig }}^{3}}{h_{\text {conv }}} .
\end{gathered}
$$

Consequently, the nonlinear differential equation and its initial condition can be expressed in the dimensionless form

$$
\begin{gathered}
\varepsilon_{1} \theta \frac{d \theta}{d \tau}+\left(1-\varepsilon_{1} \theta_{\infty}\right) \frac{d \theta}{d \tau}+\varepsilon_{2}\left(\theta^{4}-\theta_{\text {surr }}^{4}\right)+\theta-\psi-\theta_{\infty}=0 \\
\theta(0)=1 .
\end{gathered}
$$

\section{Applied Analytical Methods}

In this section two analytical methods called differential transformation method (DTM) and Boubaker polynomial expansion scheme (BPES) with their application in the problem are presented.

3.1. Differential Transformation Method (DTM). For understanding the method's concept, suppose that $x(t)$ is an analytic function in domain $D$ and $t=t_{i}$ represents any point in the domain. The function $x(t)$ is then represented by one power series whose center is located at $t_{i}$. The Taylor series expansion function of $x(t)$ is in form of

$$
x(t)=\sum_{k=0}^{\infty} \frac{\left(t-t_{i}\right)^{k}}{k !}\left[\frac{d^{k} x(t)}{d t^{k}}\right]_{t=t_{i}}, \quad \forall t \in D .
$$

The Maclaurin series of $x(t)$ can be obtained by taking $t_{i}=0$ in (11) expressed as

$$
x(t)=\sum_{k=0}^{\infty} \frac{t^{k}}{k !}\left[\frac{d^{k} x(t)}{d t^{k}}\right]_{t=0}, \quad \forall t \in D .
$$

As explained in [27], the differential transformation of the function $x(t)$ is defined as follows:

$$
X(k)=\frac{H^{k}}{k !}\left[\frac{d^{k} x(t)}{d t^{k}}\right]_{t=0},
$$

where $X(k)$ represents the transformed function and $x(t)$ is the original function. The differential spectrum of $X(k)$ is confined within the interval $t \in[0, H]$, where $H$ is a constant value. The differential inverse transformation of $X(k)$ is defined as follows:

$$
x(t)=\sum_{k=0}^{\infty}\left(\frac{t}{H}\right)^{k} X(k) .
$$

TABLE 1: Some fundamental operations of the differential transformation method.

\begin{tabular}{lc}
\hline Origin function & Transformed function \\
\hline$x(t)=\alpha f(x) \pm \beta g(t)$ & $X(k)=\alpha F(k) \pm \beta G(k)$ \\
$x(t)=\frac{d^{m} f(t)}{d t^{m}}$ & $X(k)=\frac{(k+m) ! F(k+m)}{k !} F(l) G(k-l)$ \\
$x(t)=f(t) g(t)$ & $X(k)=\delta(k-m)= \begin{cases}1, & \text { if } k=m, \\
0, & \text { if } k \neq m\end{cases}$ \\
$x(t)=t^{m}$ & $X(k)=\frac{1}{k !}$ \\
$x(t)=\exp (t)$ & $X(k)=\frac{\omega^{k}}{k !} \sin \left(\frac{k \pi}{2}+\alpha\right)$ \\
$x(t)=\sin (\omega t+\alpha)$ & $X(k)=\frac{\omega^{k}}{k !} \cos \left(\frac{k \pi}{2}+\alpha\right)$ \\
$x(t)=\cos (\omega t+\alpha)$ &
\end{tabular}

It is clear that the concept of differential transformation is based the Taylor series expansion. The values of function $X(k)$ at values of argument $k$ are referred to as discrete; that is, $X(0)$ is known as the zero discrete, $X(1)$ as the first discrete, and so forth. The more discreteness available, the more precise it is possible to restore the unknown function. The function $x(t)$ consists of the $T$-function $X(k)$, and its value is given by the sum of the $T$-functions with $(t / H)^{k}$ as its coefficient. In real applications, at the right choice of constant $H$, the larger values of argument $k$ the discrete of spectrum reduce rapidly. Some important mathematical operations performed by differential transformation method are listed in Table 1. By applying DTM from Table 1, transformed form of (9) will be

$$
\begin{aligned}
(k+1) \Theta(k+1) & \\
& +\varepsilon_{1} \sum_{l=0}^{k} \Theta(l)(k+1-l) \Theta(k+1-l) \\
& -\varepsilon_{1} \theta_{\infty}(k+1) \Theta(k+1)+\Theta(k) \\
& +\varepsilon_{2} \sum_{k_{2}=0}^{k} \sum_{k_{1}=0}^{k_{2}} \sum_{l=0}^{k_{1}} \Theta(l) \Theta\left(k_{2}-k_{1}\right) \Theta\left(k-k_{2}\right) \Theta\left(k_{1}-l\right) \\
& -\delta(k)\left(\theta_{\infty}+\varepsilon_{2} \theta_{\text {surr }}^{4}+\psi\right)
\end{aligned}
$$$$
=0 \text {, }
$$

where $\Theta$ is transformed form of $\theta$ and

$$
\delta(k)= \begin{cases}1 & k=0 \\ 0 & k \neq 1\end{cases}
$$

Transformed form of initial condition (see (10)) will be

$$
\Theta(0)=1 .
$$


TABle 2: Properties and conditions for combustion of iron particles.

\begin{tabular}{lcccrr}
\hline Particle diameter $(\mu \mathrm{m})$ & $\varepsilon_{1}$ & $\varepsilon_{2}$ & $\theta_{\infty}$ & $\theta_{\text {surr }}$ \\
\hline 20 & 0.051595 & 0.002630 & 0.98579 & 1.17647 & 0.35294 \\
60 & 0.051595 & 0.007567 & 2.83636 & 1.17647 & 0.35294 \\
100 & 0.051595 & 0.011493 & 4.30774 & 1.17647 & 0.35294 \\
\hline
\end{tabular}

For example, for an iron particle with $20 \mu \mathrm{m}$ diameter (see Table 2) solving (15) makes

$$
\begin{gathered}
\Theta(0)=1, \quad \Theta(1)=1.170326597, \\
\Theta(2)=-0.6324112173, \\
\Theta(3)=0.2462459086, \\
\Theta(4)=-0.08656001439, \ldots .
\end{gathered}
$$

By substituting DTM transformed terms of $T$ (see (18)) into (13), $\theta(\tau)$ can be determined as

$$
\begin{aligned}
\theta(\tau)= & 1+1.17033 \tau-0.632411 \tau^{2} \\
& +0.246245 \tau^{3}-0.08656 \tau^{4}+0.0324505 \tau^{5} \\
& -0.0130388 \tau^{6}+0.00504942 \tau^{7} \\
& -0.0017 \tau^{8}+0.00042358 \tau^{9} \\
& -0.114879 e-4 \tau^{10}
\end{aligned}
$$

3.2. Boubaker Polynomials Expansion Scheme (BPES). The resolution of system (9) and (10) along with boundary conditions has been achieved using the Boubaker polynomials expansion scheme BPES [7-26]. This scheme is a resolution protocol which has been successfully applied to several applied physics and mathematics problems. The BPES protocol ensures the validity of the related boundary conditions regardless of main equation features. The Boubaker polynomials expansion scheme BPES is based on the Boubaker polynomials first derivatives properties:

$$
\begin{gathered}
\left.\sum_{q=1}^{N} B_{4 q}(x)\right|_{x=0}=-2 N \neq 0, \\
\left.\sum_{q=1}^{N} B_{4 q}(x)\right|_{x=r_{q}}=0 \\
\left.\sum_{q=1}^{N} \frac{d B_{4 q}(x)}{d x}\right|_{x=0}=0 \\
\left.\sum_{q=1}^{N} \frac{d B_{4 q}(x)}{d x}\right|_{x=r_{q}}=\sum_{q=1}^{N} H_{q},
\end{gathered}
$$

$$
\text { with } \begin{aligned}
: H_{n} & =B_{4 n}^{\prime}\left(r_{n}\right) \\
& =\left(\frac{4 r_{n}\left[2-r_{n}^{2}\right] \times \sum_{q=1}^{n} B_{4 q}^{2}\left(r_{n}\right)}{B_{4(n+1)}\left(r_{n}\right)}+4 r_{n}^{3}\right)
\end{aligned}
$$

Several solutions have been proposed through the BPES in many fields such as numerical analysis [7-10], theoretical physics [10-13], mathematical algorithms [14], heat transfer [15], homodynamic [16, 17], material characterization [18], fuzzy systems modeling [19-24], and biology [25, 26].

The resolution protocol is based on setting $\theta(\tau)$ as an estimator to the $\tau$-dependent variable:

$$
\theta(\tau)=\frac{1}{2 N_{0}} \sum_{k=1}^{N_{0}} \xi_{k} \times B_{4 k}\left(\tau \times r_{k}\right)
$$

where $B_{4 k}$ are the $4 k$-order Boubaker polynomials $[7,8]$, $r_{k}$ are $B_{4 k}$ minimal positive roots, $N_{0}$ is a prefixed integer, and $\left.\xi_{k}\right|_{k=1 \cdots N_{0}}$ are unknown pondering real coefficients.

The main advantage of this formulation is the verification of boundary conditions, expressed in (9), in advance to resolution process. In fact, thanks to the properties expressed in (21)-(22), these conditions are reduced to the inherently verified linear equations:

$$
\sum_{k=1}^{N_{0}} \xi_{k}=-N_{0} .
$$

The BPES solution for (9) is obtained, according to the principles of the BPES, by determining the nonnull set of coefficients $\left.\xi_{k}\right|_{k=1 \cdots N_{0}}$ that minimize the absolute difference between left and right sides of the following equations, which follow a majoring the quadrature term within the sum:

$$
\begin{gathered}
\varepsilon_{1}\left(\frac{1}{2 N_{0}}\right)^{2} \sum_{k=1}^{N_{0}} \xi_{k} B_{4 k}(\hat{t}) \sum_{k=1}^{N_{0}} \xi_{k} r_{k} \times \frac{d B_{4 k}(\hat{t})}{d \tau} \\
+\frac{1-\varepsilon_{1} \theta_{\infty}}{2 N_{0}} \sum_{k=1}^{N_{0}} \xi_{k} r_{k} \times \frac{d B_{4 k}(\hat{t})}{d \tau} \\
+\frac{4 \varepsilon_{2}}{\left(2 N_{0}\right)^{4}} \sum_{k=1}^{N_{0}} \xi_{k} B_{4 k}(\hat{t})-\varepsilon_{2} \theta_{\text {surr }}
\end{gathered}
$$




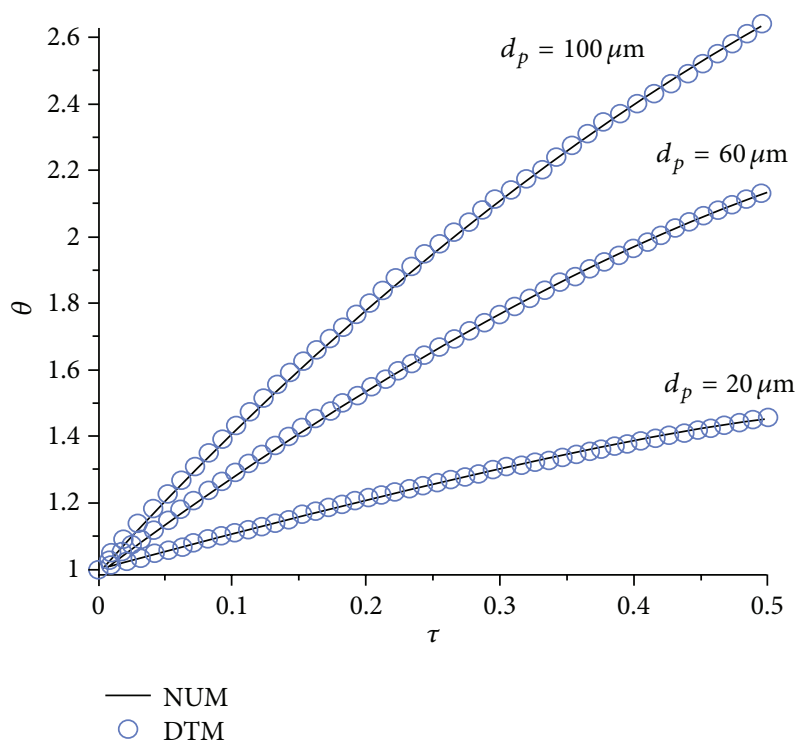

(a)

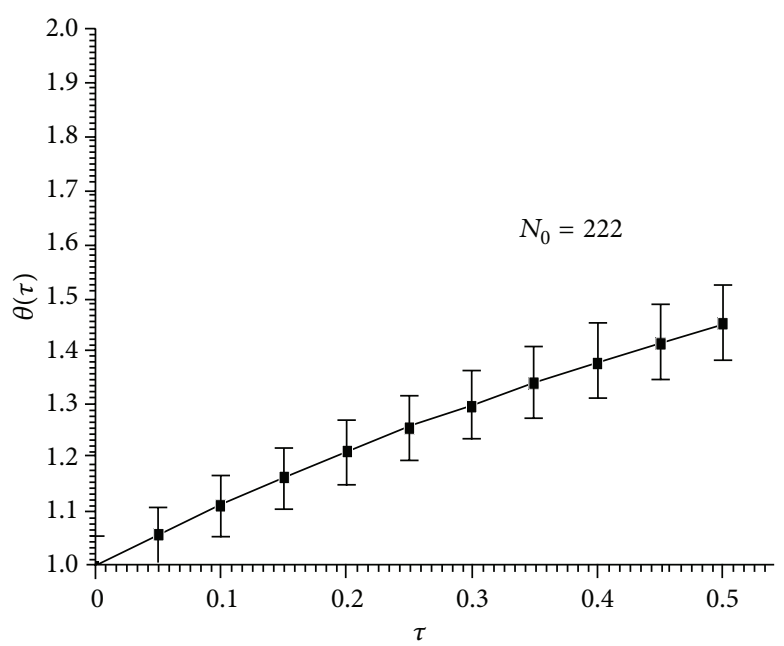

(b)

FIGURE 1: (a) Comparison between DTM and numerical method in different particle diameters (see Table 2), (b) BPES results for particle temperature for $d_{p}=20 \mu \mathrm{m}$.

TABLE 3: Comparison of DTM and BPES results with fourth-order Runge-Kutta numerical method for diameter $20 \mu \mathrm{m}$.

\begin{tabular}{|c|c|c|c|c|c|}
\hline \multirow{2}{*}{$\tau$} & \multicolumn{5}{|c|}{$\theta(\tau)$} \\
\hline & Num & DTM & BPES & $\%$ Error DTM & $\%$ Error BPES \\
\hline 0.0 & 1.00000 & 1.00000 & 1.00000000000 & - & - \\
\hline 0.05 & 1.05696555223261 & 1.056965552 & 1.05696555214 & $2.20073332404 E-8$ & $8.80293749773 E-9$ \\
\hline 0.10 & 1.11094655557423 & 1.110946451 & 1.11094651374 & $9.41307477101 E-6$ & $3.76522990441 E-6$ \\
\hline 0.15 & 1.16210940722201 & 1.162109321 & 1.16210937273 & $7.41943998047 E-6$ & $2.96777599601 E-6$ \\
\hline 0.20 & 1.21060998272779 & 1.210609952 & 1.21060997044 & $2.53820722847 E-6$ & $1.01528288038 E-6$ \\
\hline 0.25 & 1.25659429223923 & 1.256594206 & 1.25659425774 & $6.86293344734 E-6$ & $2.745173386 E-6$ \\
\hline 0.30 & 1.30019895535529 & 1.300198823 & 1.30019890241 & $1.01796182264 E-5$ & $4.0718472974 E-6$ \\
\hline 0.35 & 1.34155222687316 & 1.341552153 & 1.34155219732 & $5.50654373 E-6$ & $2.20261750193 E-6$ \\
\hline 0.40 & 1.38077485817965 & 1.380774801 & 1.38077483531 & $4.14112769994 E-6$ & $1.65645107354 E-6$ \\
\hline 0.45 & 1.41798035901816 & 1.417980211 & 1.41798029981 & $1.04386608044 E-5$ & $4.17546432177 E-6$ \\
\hline 0.50 & 1.45327532764821 & 1.453275203 & 1.45327527779 & $8.57705402442 E-6$ & $3.43082161282 E-6$ \\
\hline
\end{tabular}

$$
\begin{gathered}
+\frac{1}{2 N_{0}} \sum_{k=1}^{N_{0}} \xi_{k} B_{4 k}(\hat{t})-\psi-\theta_{\infty} \\
=0, \quad \\
\hat{t}=\tau \times r_{k} .
\end{gathered}
$$

The final solution is obtained by substituting the obtained values of the coefficients $\left.\xi_{k}\right|_{k=1 \cdots N_{0}}$ in (22).

\section{Results and Discussion}

As described in problem description section, the equation of combustion for a particle was introduced as (9) with boundary condition equation (10). Some constant parameters for this equation are introduced in Table 2 for iron particle.
For these three particle diameters, (9) is solved by DTM and BPES whose results are presented through Figures 1(a) and 1(b), respectively. As seen in these figures, both of the two analytical methods have very excellent agreement with numerical fourth-order Runge-Kutta method, and also these figures reveal that when particle diameter increases, an increase in combustion temperature due to higher energy realized in combustion is observed. The figures indicate that the temperature of iron particle (for all sizes) reaches the maximum value at the end of the burning time, and the chemical reaction, is finished, and larger particles have higher maximum temperature than that of smaller particles. This result is similar to the experimental result presented by Tang et al. [34] and analytical method by Bidabadi and Mafi [5]. Obtained data from applied methods for a special case $\left(d_{p}=20 \mu \mathrm{m}\right)$ are compared in Table 3. This table confirms that BPES has lower errors compared to DTM, but both 


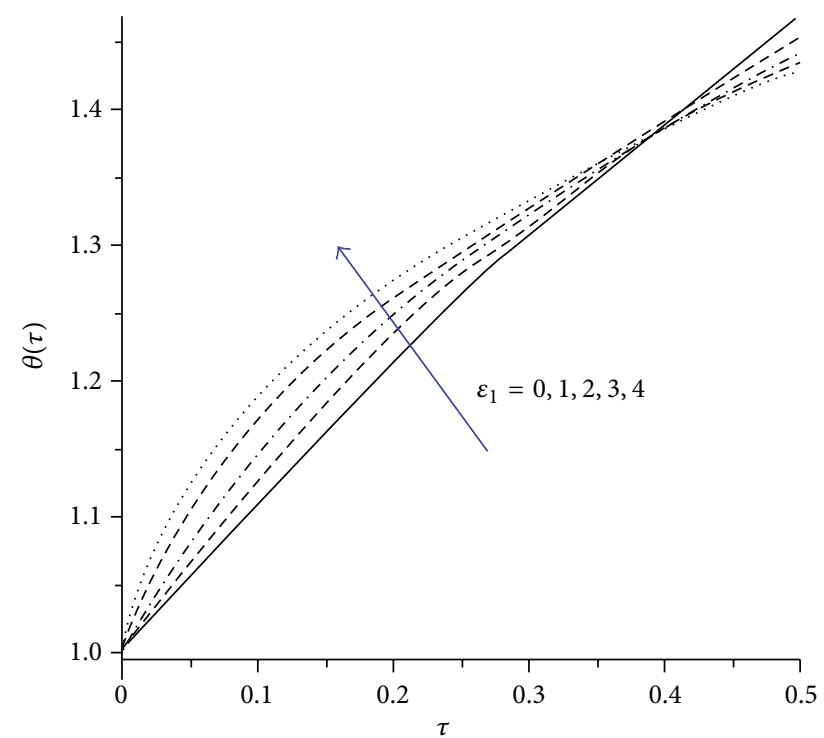

(a)

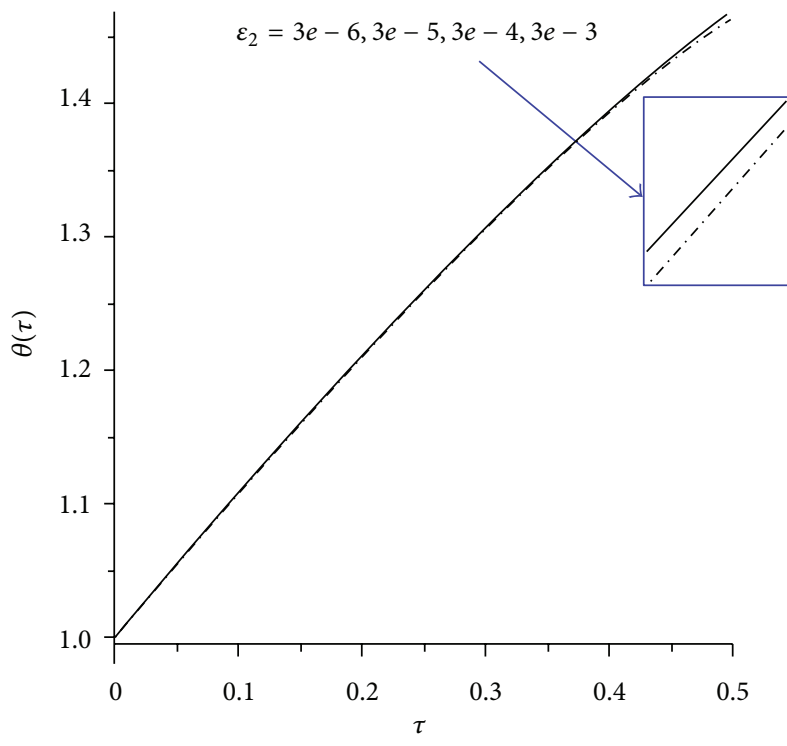

(b)

FIGURE 2: (a) Effect of $\varepsilon_{1}$ on nondimensional temperature profile for micro- and nanoparticles, (b) effect of $\varepsilon_{2}$ on nondimensional temperature profile for micro- and nanoparticles.

methods are convenient and accurate. Effects of $\varepsilon_{1}$ and $\varepsilon_{2}$ on nondimensional temperature profile for nanoparticles are shown in Figures 2(a) and 2(b), respectively. As seen, the increase in $\varepsilon_{1}$ makes an increase in temperature profile, but $\varepsilon_{2}$ makes a decrease due to the increase in radiation heat transfer term in the particle (see (8)).

An important point for particle combustion is surrounding temperature in which particle is combusted. As (2) reveals, energy inputs from the surrounding occur through radiation heat transfer, and when surrounding has higher temperature, input energy increases and consequently temperature in combustion of particle will increase. This effect is depicted in Figure 3(a). Figure 3(b) demonstrates the effect

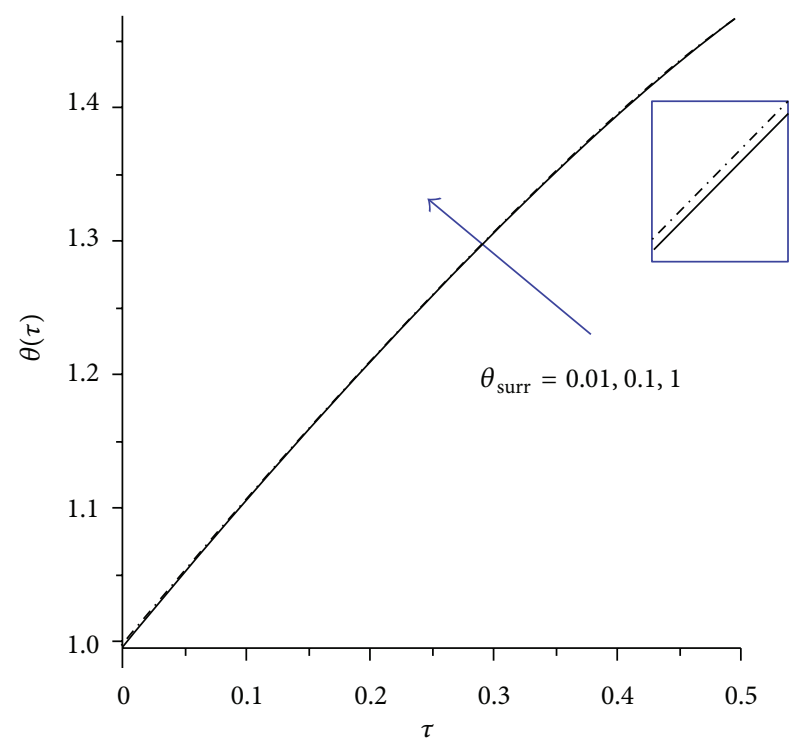

(a)

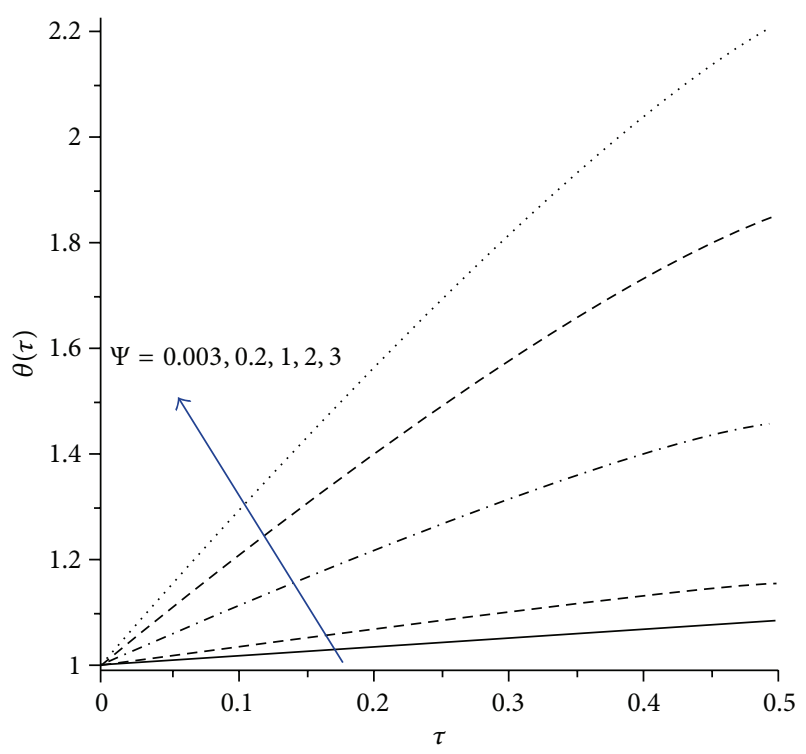

(b)

FIGURE 3: (a) Effect of surrounding temperature on nondimensional temperature profile, (b) effect of heat realized parameter $(\Psi)$ on nondimensional temperature profile.

of heat realized parameter $(\Psi)$ on temperature profile versus time. It is completely evident that by increasing the generated heat in the combustion, temperature will increase significantly.

\section{Conclusion}

In this study, the equation of temperature variation in combustion process for particles is presented. The effects of thermal radiation from the external surface of burning particle and alterations of density of iron particle with temperature are considered. Due to nonlinearity of described equation, Boubaker polynomial expansion scheme (BPES) 
and differential transformation method (DTM) have been presented in order to obtain analytical solutions. Results show that both methods have good agreement with numerical method, but BPES had lower errors than DTM. Also the effects of surrounding temperature, realized heat parameter, particle diameter, and convection and radiation heat transfer parameter on combustion temperature have been investigated.

\section{References}

[1] A. Haghiri and M. Bidabadi, "Dynamic behavior of particles across flame propagation through micro-iron dust cloud with thermal radiation effect," Fuel, vol. 90, no. 7, pp. 2413-2421, 2011.

[2] Y. Liu, J. Sun, and D. Chen, "Flame propagation in hybrid mixture of coal dust and methane," Journal of Loss Prevention in the Process Industries, vol. 20, no. 4-6, pp. 691-697, 2007.

[3] M. Bidabadi, A. Haghiri, and A. Rahbari, "Mathematical modeling of velocity and number density profiles of particles across the flame propagation through a micro-iron dust cloud," Journal of Hazardous Materials, vol. 176, no. 1-3, pp. 146-153, 2010.

[4] A. Haghiri and M. Bidabadi, "Modeling of laminar flame propagation through organic dust cloud with thermal radiation effect," International Journal of Thermal Sciences, vol. 49, no. 8, pp. 1446-1456, 2010.

[5] M. Bidabadi and M. Mafi, "Time variation of combustion temperature and burning time of a single iron particle," International Journal of Thermal Sciences, vol. 65, pp. 136-147, 2013.

[6] K. Boubaker, "The Boubaker polynomials, a new function class for solving bi-varied second order differential equations," Far East Journal of Applied Mathematics, vol. 31, pp. 299-320, 2008.

[7] M. Agida and A. S. Kumar, "A Boubaker Polynomials Expansion Scheme solution to random Love's equation in the case of a rational kernel," Electronic Journal of Theoretical Physics, vol. 7, no. 24, pp. 319-326, 2010.

[8] A. Yildirim, S. T. Mohyud-Din, and D. H. Zhang, "Analytical solutions to the pulsed Klein-Gordon equation using Modified Variational Iteration Method (MVIM) and Boubaker Polynomials Expansion Scheme (BPES)," Computers and Mathematics with Applications, vol. 59, no. 8, pp. 2473-2477, 2010.

[9] J. Ghanouchi, H. Labiadh, and K. Boubaker, "An attempt to solve the heat transfer equation in a model of pyrolysis spray using 4q-order m-Boubaker polynomials," International Journal of Heat and Technology, vol. 26, no. 1, pp. 49-53, 2008.

[10] S. Slama, J. Bessrour, K. Boubaker, and M. Bouhafs, "A dynamical model for investigation of $\mathrm{A}_{3}$ point maximal spatial evolution during resistance spot welding using Boubaker polynomials," The European Physical Journal of Applied Physics, vol. 44, no. 3, pp. 317-322, 2008.

[11] S. Slama, M. Bouhafs, and K. B. Ben Mahmoud, "A Boubaker polynomials solution to heat equation for monitoring $A_{3}$ point evolution during resistance spot welding," International Journal of Heat and Technology, vol. 26, no. 2, pp. 141-145, 2008.

[12] S. Lazzez, K. B. Ben Mahmoud, S. Abroug, F. Saadallah, and M. Amlouk, "A Boubaker Polynomials Expansion Scheme (BPES)related protocol for measuring sprayed thin films thermal characteristics," Current Applied Physics, vol. 9, no. 5, pp. 11291133, 2009.
[13] T. Ghrib, K. Boubaker, and M. Bouhafs, "Investigation of thermal diffusivity-microhardness correlation extended to surfacenitrured steel using Boubaker polynomials expansion," Modern Physics Letters B, vol. 22, no. 29, pp. 2893-2907, 2008.

[14] S. Fridjine, K. B. Ben Mahmoud, M. Amlouk, and M. Bouhafs, "A study of sulfur/selenium substitution effects on physical and mechanical properties of vacuum-grown ZnS1-xSex compounds using Boubaker Polynomials Expansion Scheme (BPES)," Journal of Alloys and Compounds, vol. 479, no. 1-2, pp. 457-461, 2009.

[15] C. Khélia, K. Boubaker, T. Ben Nasrallah, M. Amlouk, and S. Belgacem, "Morphological and thermal properties of $\beta$-SnS2 sprayed thin films using Boubaker polynomials expansion," Journal of Alloys and Compounds, vol. 477, no. 1-2, pp. 461-467, 2009.

[16] K. B. Ben Mahmoud and M. Amlouk, “The 3D AmloukBoubaker expansivity-energy gap-Vickers hardness abacus: a new tool for optimizing semiconductor thin film materials," Materials Letters, vol. 63, no. 12, pp. 991-994, 2009.

[17] M. Dada, O. B. Awojoyogbe, and K. Boubaker, "Heat transfer spray model: an improved theoretical thermal time-response to uniform layers deposit using Bessel and Boubaker polynomials," Current Applied Physics, vol. 9, no. 3, pp. 622-624, 2009.

[18] S. Tabatabaei, T. Zhao, O. Awojoyogbe, and F. Moses, "Cutoff cooling velocity profiling inside a keyhole model using the Boubaker Polynomials Expansion Scheme," International Journal of Heat and Mass Transfer, vol. 45, no. 10, pp. 1247-1251, 2009.

[19] A. Belhadj, J. Bessrour, M. Bouhafs, and L. Barrallier, "Experimental and theoretical cooling velocity profile inside laser welded metals using keyhole approximation and Boubaker polynomials expansion," Journal of Thermal Analysis and Calorimetry, vol. 97, no. 3, pp. 911-915, 2009.

[20] A. Belhadj, O. F. Onyango, and N. Rozibaeva, "Boubaker polynomials expansion scheme-related heat transfer investigation inside keyhole model," Journal of Thermophysics and Heat Transfer, vol. 23, no. 3, pp. 639-640, 2009.

[21] P. Barry and A. Hennessy, "Meixner-type results for Riordan arrays and associated integer sequences," Journal of Integer Sequences, vol. 13, no. 9, pp. 1-34, 2010.

[22] A. S. Kumar, "An analytical solution to applied mathematicsrelated Love's equation using the Boubaker Polynomials Expansion Scheme," Journal of the Franklin Institute, vol. 347, no. 9, pp. 1755-1761, 2010.

[23] S. Fridjine and M. Amlouk, "A new parameter: an abacus for optimizing PVT hybrid solar device functional materials using the Boubaker Polynomials Expansion Scheme," Modern Physics Letters B, vol. 23, no. 17, pp. 2179-2191, 2009.

[24] M. Benhaliliba, C. E. Benouis, K. Boubaker, M. Amlouk, and A. Amlouk, "A new guide to thermally optimized doped oxides monolayer spray-grown solar cells: the Amlouk-Boubaker optothermal expansivity $\psi \mathrm{ab}$," in Solar Cells-New Aspects and Solutions, L. A. Kosyachenko, Ed., pp. 27-41, InTech, 2011.

[25] A. Milgram, "The stability of the Boubaker Polynomials Expansion Scheme (BPES)-based solution to Lotka-Volterra problem," Journal of Theoretical Biology, vol. 271, no. 1, pp. 157-158, 2011.

[26] H. Rahmanov, "A solution to the non linear Korteweg-De-Vries equation in the particular case dispersion-adsorption problem in porous media using the spectral Boubaker Polynomials Expansion Scheme (BPES)," Studies in Nonlinear Sciences, vol. 2, no. 1, pp. 46-49, 2011. 
[27] K. Zhou, Differential Transformation and Its Applications for Electrical CircuIts, Huazhong University Press, Wuhan, China, 1986.

[28] C. K. Chen and S. H. Ho, "Solving partial differential equations by two-dimensional differential transform method," Applied Mathematics and Computation, vol. 106, no. 2-3, pp. 171-179, 1999.

[29] F. Ayaz, "Solutions of the system of differential equations by differential transform method," Applied Mathematics and Computation, vol. 147, no. 2, pp. 547-567, 2004.

[30] I. H. A.-H. Hassan, "Comparison differential transformation technique with adomian decomposition method for linear and nonlinear initial value problems," Chaos, Solitons and Fractals, vol. 36, no. 1, pp. 53-65, 2008.

[31] M.-J. Jang, C.-L. Chen, and Y.-C. Liu, "Two-dimensional differential transform for partial differential equations," Applied Mathematics and Computation, vol. 121, no. 2-3, pp. 261-270, 2001.

[32] A. A. Joneidi, D. D. Ganji, and M. Babaelahi, "Differential transformation method to determine fin efficiency of convective straight fins with temperature dependent thermal conductivity," International Communications in Heat and Mass Transfer, vol. 36, no. 7, pp. 757-762, 2009.

[33] M. Momeni, N. Jamshidi, A. Barari, and G. Domairry, "Numerical analysis of flow and heat transfer of a viscoelastic fluid over a stretching sheet," International Journal of Numerical Methods for Heat and Fluid Flow, vol. 21, no. 2, pp. 206-218, 2011.

[34] F. D. Tang, S. Goroshin, and A. J. Higgins, "Modes of particle combustion in iron dust flames," Proceedings of the Combustion Institute, vol. 33, no. 2, pp. 1975-1982, 2011. 

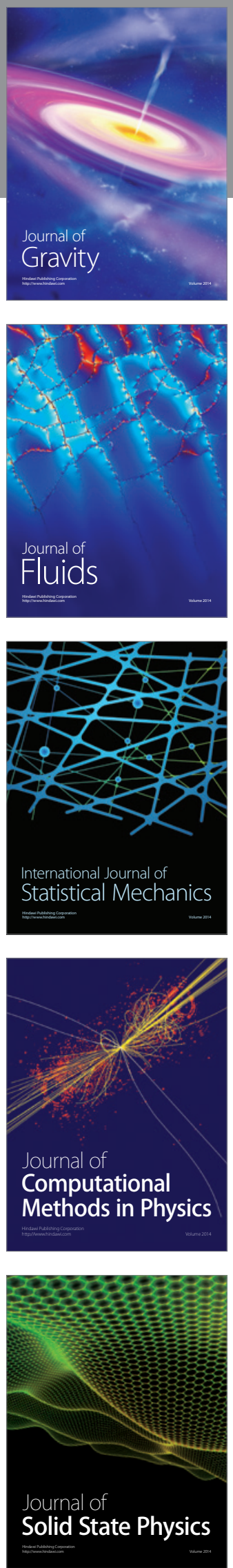

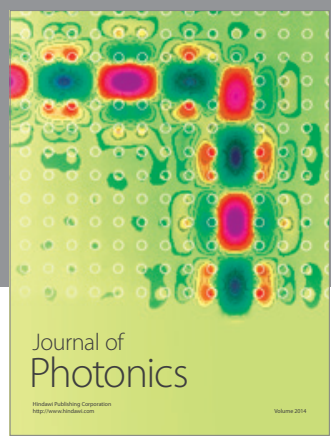

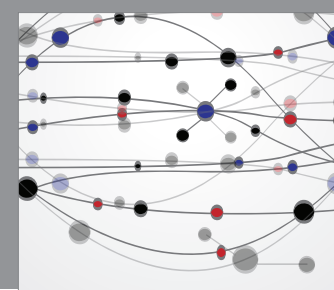

The Scientific World Journal

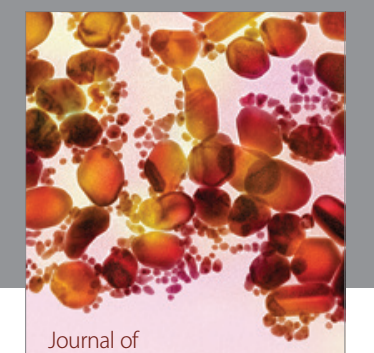

Soft Matter
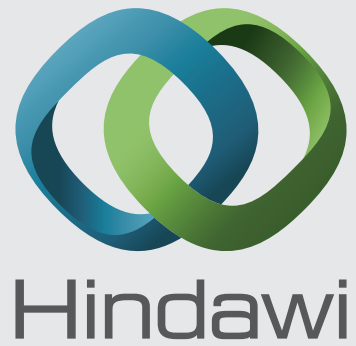

Submit your manuscripts at

http://www.hindawi.com
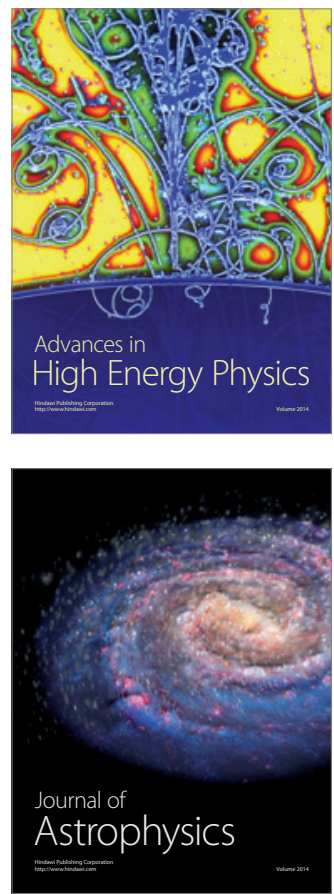
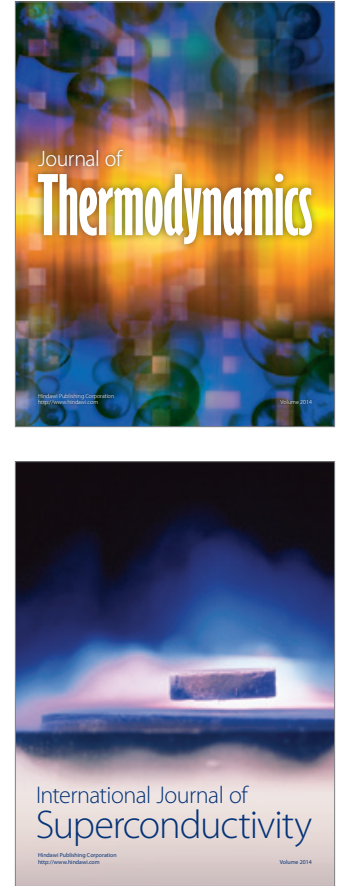
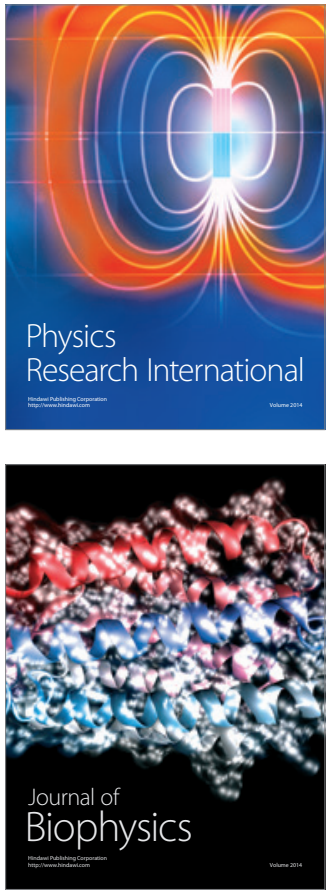
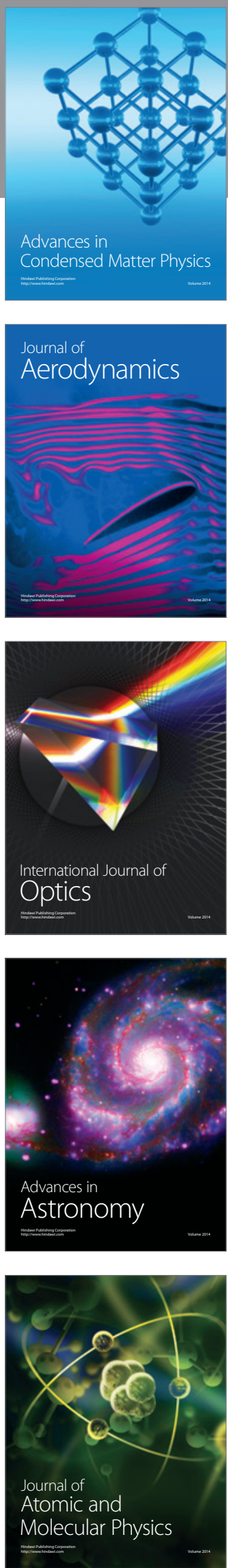